\title{
Stabilization and destabilization in non-conservative gyroscopic systems
}

\author{
Oleg N. Kirillov ${ }^{1, *}$ \\ ${ }^{1}$ Institute of Mechanics, Moscow State Lomonosov University, Michurinskii pr. 1, 119192 Moscow, Russia.
}

\begin{abstract}
Stability of a linear autonomous non-conservative system in presence of potential, gyroscopic, dissipative, and nonconservative positional forces is studied. The cases when the non-conservative system is close to a gyroscopic system or to a circulatory one, are examined. It is known that the marginal stability of gyroscopic and circulatory systems can be destroyed or improved up to asymptotic stability due to action of small non-conservative positional and velocity-dependent forces. The present contribution shows that in both cases the boundary of the asymptotic stability domain of the perturbed system possesses singularities such as "Dihedral angle" and "Whitney umbrella" that govern stabilization and destabilization. Approximations of the stability boundary near the singularities and estimates of the critical gyroscopic and circulatory parameters are found in an analytic form. In case of two degrees of freedom these estimates are obtained in terms of the invariants of matrices of the system. As an example, the asymptotic stability domain of the modified Maxwell-Bloch equations is investigated with an application to the stability problems of gyroscopic systems with stationary and rotating damping, such as the Crandall gyropendulum, tippe top and Jellet's egg. An instability mechanism in a system with two degrees of freedom, originating after discretization of models of a rotating disc in frictional contact and possessing the spectral mesh in the plane 'frequency' versus 'angular velocity', is described in detail and its role in the disc brake squeal problem is discussed.
\end{abstract}

() 2007 WILEY-VCH Verlag GmbH \& Co. KGaA, Weinheim

\section{Bifurcation of the domain of asymptotic stability, singularities on its boundary, and the critical movement of eigenvalues}

Consider an autonomous non-conservative system described by a linear differential equation of second order

$$
\ddot{\mathbf{x}}+(\Omega \mathbf{G}+\delta \mathbf{D}) \dot{\mathbf{x}}+(\mathbf{K}+\nu \mathbf{N}) \mathbf{x}=0,
$$

where dot denotes time differentiation, $\mathbf{x} \in \mathbb{R}^{m}$, and real matrix $\mathbf{K}=\mathbf{K}^{T}$ corresponds to potential forces. Real matrices $\mathbf{D}=\mathbf{D}^{T}, \mathbf{G}=-\mathbf{G}^{T}$, and $\mathbf{N}=-\mathbf{N}^{T}$ are related to dissipative (damping), gyroscopic, and non-conservative positional (circulatory) forces with magnitudes controlled by scaling factors $\delta, \Omega$, and $\nu$ respectively. A circulatory system is obtained from (1) by neglecting velocity-dependent forces

$$
\ddot{\mathbf{x}}+(\mathbf{K}+\nu \mathbf{N}) \mathbf{x}=0,
$$

while a gyroscopic one has no damping and non-conservative positional forces

$$
\ddot{\mathbf{x}}+\Omega \mathbf{G} \dot{\mathbf{x}}+\mathbf{K x}=0 .
$$

The circulatory system (2) possesses a reversible symmetry while the gyroscopic system (3) possesses the Hamiltonian one, which is seen after transformation of equation (1) to the Cauchy form $\dot{\mathbf{y}}=\mathbf{A y}$. As a consequence, $\operatorname{det}(\mathbf{A}-\lambda \mathbf{I})=$ $\operatorname{det}(\mathbf{A}+\lambda \mathbf{I})$, so that the eigenvalues of systems (2) and (3) appear in pairs $(-\lambda, \lambda)$. Hence, the equilibrium of the circulatory system as well as of the gyroscopic one is either unstable or all its eigenvalues lie on the imaginary axis of the complex plane implying marginal stability, if they are semi-simple. In the presence of all the four forces the Hamiltonian and reversible symmetries are broken and the marginal stability is generally destroyed. Instead, system (1) can be asymptotically stable if its characteristic polynomial $P(\lambda)=\operatorname{det}\left(\mathbf{I} \lambda^{2}+(\Omega \mathbf{G}+\delta \mathbf{D}) \lambda+\mathbf{K}+\nu \mathbf{N}\right)$, satisfies the criterion of Routh and Hurwitz. The most interesting for many applications is the situation when system (1) is close either to circulatory system (2) with $\delta, \Omega \ll \nu$ (imperfect reversible system) or to gyroscopic system (3) with $\delta, \nu \ll \Omega$ (imperfect Hamiltonian system). Furthermore, the effect of small damping and gyroscopic forces on the stability of circulatory systems as well as the effect of small damping and non-conservative positional forces on the stability of gyroscopic systems are regarded as paradoxical, since the stability properties are extremely sensitive to the choice of the perturbation, and the balance of forces resulting in the asymptotic stability is not evident, as it happens in such phenomena as "tippe top inversion", "rising egg", and the onset of frictioninduced oscillation in the squealing brake and the singing wine glass [1-7].

In general, an effective tool for analytical study of the stability of imperfect Hamiltonian and reversible systems is a perturbation theory for multiple eigenvalues [1-7]. For example, the critical value for the onset of gyroscopic stabilization in the presence of small dissipation and non-conservative forces is given by this approach as

$$
\Omega_{c r}(\delta, \nu)=\Omega_{0}+\frac{n_{1}^{2}\left(\nu-\beta_{0} \delta\right)^{2}}{\mu^{2}\left(\omega_{0} d_{2}-\beta_{0} n_{2}-d_{1}\right)^{2} \delta^{2}} \geq \Omega_{0}, \quad \beta_{0}=-i \omega_{0} \frac{\mathbf{u}_{0}^{*} \mathbf{D} \mathbf{u}_{0}}{\mathbf{u}_{0}^{*} \mathbf{N} \mathbf{u}_{0}},
$$

\footnotetext{
* Corresponding author E-mail: kirillov@imec.msu.ru, Phone: +7495939 1255, Fax: +74959390165
} 
a)

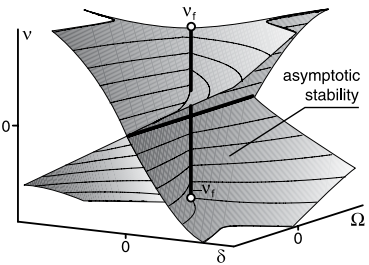

b)

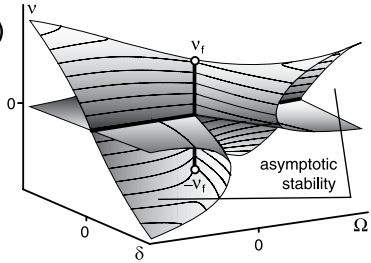

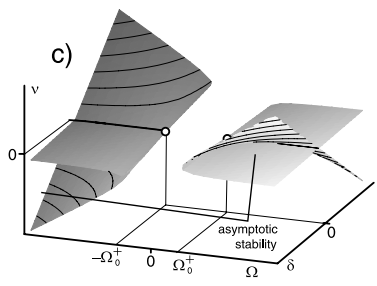

d)

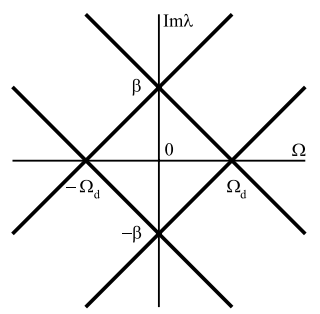

h)

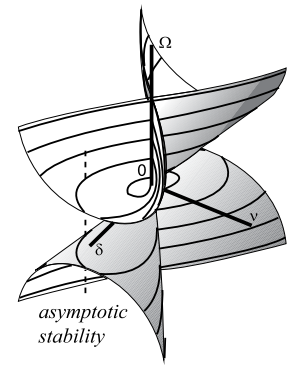

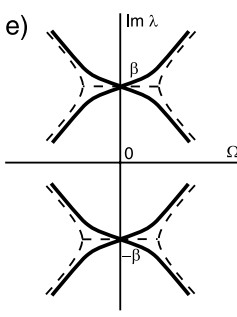

i)

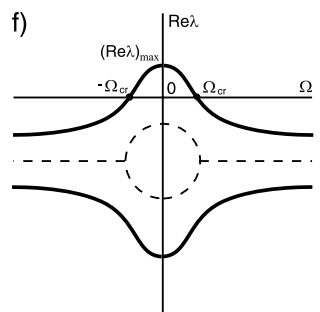

g)
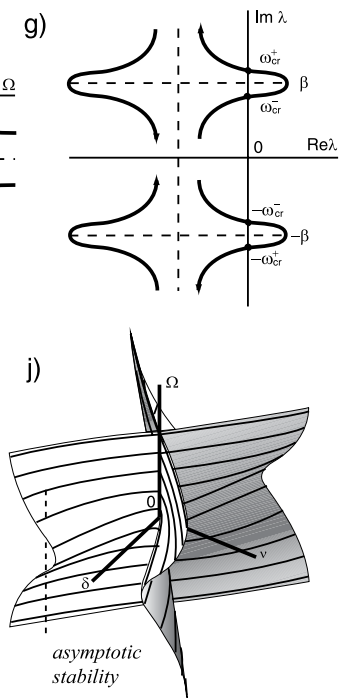

Fig. 1 Asymptotic stability domain for the system (1) with 2 d.o.f. when $\operatorname{tr} \mathbf{K}>0$, det $\mathbf{K}>0$ and the sign of the left hand side in (7) is positive (a) or negative (b) and when $\mathbf{K}<0$ (c); critical eigenvalue movement for the system (8) with 2 d.o.f. when $\delta=\nu=0$ (d), and when $\delta \neq 0$ and $\nu \neq 0$ (e)-(g); bifurcation of the domain of asymptotic stability of the system (8) with 2 d.o.f. when det $\mathbf{D}$ is continuously changing from the positive values $(h)$ to zero (i) and negative ones $(j)$.

where $\Omega_{0}$ is the onset of the gyroscopic stabilization of system (3) and the real scalars $d_{1}, d_{2}, n_{1}$, and $n_{2}$ are calculated with the use of the generalized eigenvectors of the double eigenvalue $\lambda=i \omega_{0}$ of system (3) at $\Omega=\Omega_{0}$ [7]

$$
d_{1}=\operatorname{Re}\left(\mathbf{u}_{0}^{*} \mathbf{D} \mathbf{u}_{0}\right), \quad d_{2}=\operatorname{Im}\left(\mathbf{u}_{0}^{*} \mathbf{D} \mathbf{u}_{1}-\mathbf{u}_{1}^{*} \mathbf{D} \mathbf{u}_{0}\right), \quad n_{1}=\operatorname{Im}\left(\mathbf{u}_{0}^{*} \mathbf{N} \mathbf{u}_{0}\right), \quad n_{2}=\operatorname{Re}\left(\mathbf{u}_{0}^{*} \mathbf{N} \mathbf{u}_{1}-\mathbf{u}_{1}^{*} \mathbf{N} \mathbf{u}_{0}\right) .
$$

In case of the two d.o.f. $\mu=\Omega_{0} / 2$ and the formulas (4) are expressed directly by means of the matrices of the system (1) [3,4]

$$
\Omega_{c r}(\nu, \delta)=\Omega_{0}+2 \Omega_{0}\left(\omega_{0} \operatorname{Tr} \mathbf{D}\right)^{-2}\left(\nu / \delta-\beta_{0}\right)^{2}, \quad \beta_{0}=\left(2 \Omega_{0}\right)^{-1} \operatorname{Tr}\left[\left(\Omega_{0}^{2}-\omega_{0}^{2}\right) \mathbf{D}+\mathbf{K D}\right] .
$$

The equations (4) and (6) describe a surface with the singularity Whitney umbrella [1-7], which is seen in Fig. 1 (a)-(c), where the domain of asymptotic stability of the system (1) with 2 d.o.f. is shown. The domain bifurcates when det $\mathbf{D}$ passes through the threshold

$$
\operatorname{det} \mathbf{D}+\left(2 \nu_{f}\right)^{-2}\left(k_{12}\left(d_{22}-d_{11}\right)-d_{12}\left(k_{22}-k_{11}\right)\right)^{2}=0 ; \quad \nu_{f}=\sqrt{(\operatorname{tr} \mathbf{K} / 2)^{2}-\operatorname{det} \mathbf{K}} .
$$

A special case of the system (1), occurring, for example, in the modeling of the squealing disk brake [6]

$$
\ddot{\mathbf{x}}+(2 \Omega \mathbf{G}+\delta \mathbf{D}) \dot{\mathbf{x}}+\left(\left(\beta^{2}-\Omega^{2}\right) \mathbf{I}+\nu \mathbf{N}\right) \mathbf{x}=0,
$$

can be treated by the same method. Complicated singularities existing on the stability boundary of the system (8) (see Fig. 1 (h)-(j)) are responsible for the excursions of the eigenvalues to the right in the complex plane, causing the subcritical flutter instability, which is the reason for self-excited oscillations of a disc brake (squeal) [6].

Acknowledgements The work has been partially supported by the Alexander von Humboldt Foundation.

\section{References}

[1] O.N. Kirillov, Destabilization paradox. Dokl. Phys. 49(4), 239-245 (2004).

[2] O.N. Kirillov, A theory of the destabilization paradox in non-conservative systems. Acta Mech. 174(3-4), 145-166 (2005).

[3] O.N. Kirillov, Gyroscopic stabilization of non-conservative systems. Phys. Lett. A. 359(3), 204-210. (2006).

[4] O.N. Kirillov, Destabilization paradox due to breaking the Hamiltonian and reversible symmetry. Int. J. Non-Lin. Mech. 42(1), 71-87 (2007).

[5] O.N. Kirillov, On the stability of nonconservative systems with small dissipation. J. of Math. Sciences. 145(5), 5260-5270 (2007).

[6] O.N. Kirillov, Subcritical flutter in acoustics of friction. Proc. Roy. Soc. A (submitted), [arXiv:0708.0967v1 [math-ph] 7 Aug 2007.]

[7] O.N. Kirillov, Gyroscopic stabilization in the presence of nonconservative forces. Dokl. Math. 76(2), 780-785. (2007). 